\title{
REVIEWER'S COMMENTS ON ARTICLE 3
}

\section{Hypoglossal-facial nerve side-to-end anastomosis for preservation of hypoglossal function: results of delayed treatment with a new technique. Yutaka Sawamura and Hiroshi Abe}

\section{By William E. Hitselberger, M.D., Los Angeles, California}

In my experience with at least 100 hypoglossal-facial nerve anastomoses, "hemiglossal dysfunction" has not been a major complaint in my patients. The restoration of mobility of facial musculature usually compensates for the deficits the authors mention.

The hypoglossal nerve normally has a larger diameter than the facial nerve and this difference is accentuated in the situation in which there is proximal interruption of the facial nerve. This leads to redundancy and waste in the use of hypoglossal "fire power." Thus, the authors' attempt to preserve hypoglossal function is both laudable and practical.

I take some issue with the implication in the paper that 24 months after injury is the cut-off point for a successful anastomosis. I do not think the outer limit has been established, and to press for the operation within 24 months could conceivably result in discounting the normal recovery of the facial nerve, which of course will give a far better result. Significantly, in the authors' own series of four patients, the duration of the facial paralysis was greater than 24 months in every case, and in the third patient it was 46 months.

The authors describe a technique in which the hypoglossal nerve is not split for any distance, which further enhances the integrity of this nerve and reduces the degree of hemiatrophy of the tongue.

Tension at the anastomotic site can be a source of disruption to the graft. This was not a problem in the cases presented here. To further reduce the tension at the anastomotic site, the posterior belly of the digastric muscle can be sectioned because it is not functional from the start.

Although the series presented is small, the technique described certainly represents an advance in this kind of surgery. As the experience of many surgeons using this technique increases, its true value will become apparent. 